Article

\title{
On Eccentric Topological Indices Based on Edges of Zero Divisor Graphs
}

\author{
Ali N. A. Koam ${ }^{1}\left(\mathbb{D}\right.$, Ali Ahmad ${ }^{2, *}$ and Azeem Haider ${ }^{1}$ (D) \\ 1 Department of Mathematics, College of Science, Jazan University, New Campus, Jazan 2097, Saudi Arabia \\ 2 College of Computer Science and Information Technology, Jazan University, Jazan 45142, Saudi Arabia \\ * Correspondence: ahmadsms@gmail.com
}

Received: 18 June 2019; Accepted: 9 July 2019; Published: 12 July 2019

check for updates

\begin{abstract}
This article is devoted to the determination of edge-based eccentric topological indices of a zero divisor graph of some algebraic structures. In particular, we computed the first Zagreb eccentricity index, third Zagreb eccentricity index, geometric-arithmetic eccentricity index, atom-bond connectivity eccentricity index and a fourth type of eccentric harmonic index for zero divisor graphs associated with a class of finite commutative rings.
\end{abstract}

Keywords: eccentricity; topological index; zero divisor graphs; commutative ring

MSC: 05C12; 05C10; 05C90

\section{Introduction}

Algebraic structures have been studied for their close affiliation with representation theory and number theory, and they have been extensively studied in combinatorics [1,2]. In addition to the extensive theoretical research in these areas, finite rings and fields have received attention for their applications to cryptography and coding theory.

A foundational role of molecular descriptors is to consider molecules as real bodies and transform them into numbers, which enables mathematics to play a key role in chemistry, pharmaceutical sciences, environmental protection, quality control and health research. These molecular descriptors are graph invariants and are usually known as topological indices. In graph theory, by using topological indices we correlate various characteristics of a chemical structure in order to characterize it. These days there is an area of research devoted to computing topological indices for various structures.

In mathematical chemistry, a molecular or chemical graph is a presentation of the structural formula of a chemical compound in the form of graph structures. A chemical graph is a labeled graph with corresponding vertices representing the atoms of the compound and edges representing their chemical bonds. A topological index associated with a chemical graph is a numeric value which remains invariant under the graph automorphism and it also characterizes the topology of a chemical graph. Hence, these indices have a variety of applications in chemistry as well as in pharmaceutical science and in the structures of nanotubes [3,4]. For graph theory, topological indices are classified as "topological indices based on degrees" [5-8], "topological indices based on distances" and "topological indices based on eccentricities" of graphs. Some well-known topological indices based on the degrees of a graph are the Randi connectivity index, Zagreb indices, Harmonic index, atom bond connectivity, geometric arithmetic index and so forth, and the Wiener index, Hosaya index and Estrada index are distance based topological indices $[9,10]$. Similarly, eccentricity based topological indices include the Zagreb eccentricity index [11,12], geometric-arithmetic eccentricity index [13], connectivity of atomic bond eccentricity index [14] and the fourth type of eccentric harmonic index [15,16]. Topological 
indices lay the criteria for the growth of chemical structures and how mathematical operations on the graphs, with the relief of topological indices, can extend multidisciplinary research.

\section{Motivation behind Topological Indices}

An association between the stability of linear alkanes and the branched alkanes is studied through the $A B C$ index. This index is also used for the computation of strain energy for cyclo alkanes $[17,18]$. In order to correlate certain physico-chemical characteristics, the GA index has more efficient predictive power than does the Randic connectivity index $[19,20]$. The Zagreb indices (first and second) are powerful tools for the computation of total p-electron energy of the molecules within a particular approximate expression [21]. The topological indices that are based on degrees are more useful for examining the chemical characteristics of distinct molecular structures. These applications provide a motivation to study topological indices that are eccentricity based. The topological indices based on eccentricity are useful for the assessment of the toxicological, physico-chemical and pharmacological characteristics of a compound through the structure of its molecules. The study of the QSAR (quantitative structure activity relationship) is known for this sort of analysis [22]. In order to obtain further details on the applications of Topological indices see References [23,24].

\section{Methods}

In order to obtain our intended outcomes we used combinatorial tools such as vertex partition, edge partition, eccentricity, eccentricity counting for each degree and other graph theoretical methods with some analytic techniques $[23,25]$. Moreover, we use $C^{++}$for the verification of degree and their corresponding eccentricity.

\section{Definitions and Notations}

Let $G$ be a connected graph with vertex set $V(G)$ and edge set $E(G)$. We associate with each graph $G$ a numerical value, called the topological index (descriptor). This index remains invariant under graph automorphisms. The number of edges adjacent to a vertex $v$ is called the degree of $v$ which is denoted by $d(v)$ and the corresponding maximum and minimum degree of a vertex in a graph $G$ is denoted by $\Delta(G)$ and $\delta(G)$ respectively. The $d(u, v)$ denotes the distance between any two vertices $u$ and $v$ which is defined as the number of edges in the shortest path between the vertices $u$ and $v$. The eccentricity of a vertex $v$ in a graph $G$ is defined as:

$$
\varepsilon_{v}=\max \{d(v, u): u \in V(G)\} .
$$

We study general topological invariant $T(G)$ based on the eccentricity of vertices in graph $G$.

$$
T(G)=\sum_{u v \in E(G)} \phi\left(\epsilon_{u}, \epsilon_{v}\right)
$$

where $\phi\left(\epsilon_{u}, \epsilon_{v}\right)=\phi\left(\epsilon_{v}, \epsilon_{u}\right)$ is a real function of $\epsilon_{u}$ and $\epsilon_{v}$.

- If $\phi\left(\epsilon_{u}, \epsilon_{v}\right)=\left(\epsilon_{u}+\epsilon_{v}\right)^{\beta}$, where $\beta \neq 0$ is a real number, then $\phi(G)$ is the first Zagreb eccentricity index if $\beta=1[11,12]$.

- If $\phi\left(\epsilon_{u}, \epsilon_{v}\right)=\left(\epsilon_{u} \times \epsilon_{v}\right)^{\alpha}$, where $\alpha \neq 0$ is a real number, then $\phi(G)$ is the third Zagreb eccentricity index if $\alpha=1[11,12]$.

- If $\phi\left(\epsilon_{u}, \epsilon_{v}\right)=\frac{2 \sqrt{\epsilon_{u} \times \epsilon_{v}}}{\epsilon_{u}+\epsilon_{v}}$, we obtain the geometric-arithmetic eccentricity index $G A_{4}(G)$ [13].

- If $\phi\left(\epsilon_{u}, \epsilon_{v}\right)=\sqrt{\frac{\epsilon_{u}+\epsilon_{v}-2}{\epsilon_{u} \times \epsilon_{v}}}$, we obtain the atom-bond connectivity eccentricity index $A B C_{5}(G)$ [14].

- If $\phi\left(\epsilon_{u}, \epsilon_{v}\right)=\frac{2}{\epsilon_{u}+\epsilon_{v}}$, we obtain the fourth type of eccentric harmonic index $H_{4}(G)[15,16]$.

A commutative ring $R$ with identity a non-zero element $x \in R$ is called a zero divisor if there exists another non zero element $y \in R$ such that $x . y=0$ in $R$. The set of all zero divisors in $R$ is denoted 
by $Z(R)$. By considering a finite commutative ring $R$, we associate a zero divisor graph $G(R)$ such that if $x_{1}, x_{2} \in Z(R)=V(G(R))$ and $\left(x_{1}, x_{2}\right) \in E(G(R))$ if and only if $x_{1} \cdot x_{2}=0$. The notion of the zero divisor graph was introduced by Beck [2] and later for any commutative ring $R$, Anderson and Livingston, in Reference [26], proved that the zero divisor graph $G(R)$ is a connected graph. Due to broad interest in parameters associated with graphs and rings, several authors investigated the associated identities of zero-divisor for commutative rings, see for example References [23,27,28].

\section{Main Results}

For positive integers $a, b \in \mathbb{Z}^{+}$, we consider $G(R)$ to be a zero divisor graph of commutative ring $R=\mathbb{Z}_{a} \times \mathbb{Z}_{b}$ whose vertex set is $V(G(R))$ and edge set is $E(G(R))$. Then

$$
V(G(R))=\left\{(x, y) \in R: x \in Z\left(\mathbb{Z}_{a}\right) \cup\{0\} \text { or } y \in Z\left(\mathbb{Z}_{b}\right) \cup\{0\}\right\} \backslash\{(0,0)\}
$$

and

$$
E(G(R))=\left\{\left(\left(x_{1}, y_{1}\right),\left(x_{2}, y_{2}\right)\right) \in V(G(R)) \times V(G(R)):\left(x_{1} x_{2}, y_{1} y_{2}\right)=(0,0) \text { in } R\right\} .
$$

This section is devoted on the discussion of eccentric topological indices based on the edges of zero divisor graphs for rings $\mathbb{Z}_{a} \times \mathbb{Z}_{b}$ with $a=p_{1} p_{2}, p^{2}$ and $b=q^{2}$, where $p_{1}, p_{2}, p$ and $q$ are prime numbers.

\subsection{Case 1: $\mathbb{Z}_{p_{1} p_{2}} \times \mathbb{Z}_{q^{2}}$}

Let $\Gamma_{R}$ be a zero divisor graph of commutative ring $R=\mathbb{Z}_{p_{1} p_{2}} \times \mathbb{Z}_{q^{2}}$, where $p_{1}, p_{2}, q$ are prime numbers with $p_{1} \neq p_{2}$, in this subsection we discuss the eccentric topological indices for $\Gamma_{R}$.

Theorem 1. Let $\Gamma_{R}$ be the zero divisor graph of commutative ring $R=\mathbb{Z}_{p_{1} p_{2}} \times \mathbb{Z}_{q^{2}}$, where $p_{1}, p_{2}, q$ are prime numbers with $p_{1} \neq p_{2}$. Then $\left|V\left(\Gamma_{R}\right)\right|=q^{2}\left(p_{2}+p_{1}-1\right)+q\left(p_{2}-1\right)\left(p_{1}-1\right)-1$ and $\left|E\left(\Gamma_{R}\right)\right|=$ $q^{2}\left(5 p_{1} p_{2}-3 p_{1}-3 p_{2}+\frac{3}{2}\right)+q\left(-4 p_{1} p_{2}+2 p_{1}+2 p_{2}-\frac{3}{2}\right)+1$.

Proof. Let $(u, v) \in V\left(\Gamma_{R}\right)$. Then we have following cases:

1. If $u=0$ and for any $v \in \mathbb{Z}_{q^{2}}$ with $v \neq k q, 0 \leq k \leq q-1$, then each $(0, v)$ of this type is connected to the vertices of type $\left(u^{\prime}, 0\right)$ for all $u^{\prime} \in \mathbb{Z}_{p_{1} p_{2}} \backslash\{0\}$. Hence the degree of each such vertex is $p_{1} p_{2}-1$. Similarly if $u \in \mathbb{Z}_{p_{1} p_{2}} \backslash\left\{0, t_{1} p_{1}, t_{2} p_{2}\right.$ with $\left.1 \leq t_{1} \leq p_{2}-1,1 \leq t_{2} \leq p_{1}-1\right\}$ and $v=0$, then the degree of each such vertex is $q^{2}-1$.

2. If $u=0$ and $v=k q, 1 \leq k \leq q-1$, then each such vertex $(0, v)$ is connected to the vertices of types $\left(u_{1}, 0\right),\left(0, v^{\prime}\right),\left(u_{2}, v^{\prime}\right),\left(u_{3}, v^{\prime}\right)$ and $\left(u_{4}, v^{\prime}\right)$ for every $u_{1} \in \mathbb{Z}_{p_{1} p_{2}} \backslash\{0\}, u_{2}=t_{1} p_{1}, 1 \leq t_{1} \leq p_{2}-1$, $u_{3}=t_{2} p_{2}, 1 \leq t_{2} \leq p_{1}-1, u_{4} \neq t_{1} p_{1}, 0 \leq t_{1} \leq p_{2}-1, u_{4} \neq t_{2} p_{2}, 0 \leq t_{2} \leq p_{1}-1$ and $v^{\prime}=t q, 1 \leq k \leq q-1$ with $t \neq k$. Hence the degree of each such vertex is $p_{1} p_{2}-1+q-2+$ $\left(p_{1}-1\right)(q-1)+\left(p_{2}-1\right)(q-1)+\left(p_{2}-1\right)\left(p_{1}-1\right)(q-1)=p_{1} p_{2}-2+\left(p_{2}+p_{1}-1\right)(q-1)+$ $\left(p_{2}-1\right)\left(p_{1}-1\right)(q-1)=p_{1} p_{2} q-2$.

3. If $u=t_{1} p_{1}, 1 \leq t_{1} \leq p_{2}-1$ and $v=0$, then each such vertex $(u, 0)$ is connected to the vertices of type $\left(0, v_{1}\right),\left(u^{\prime}, 0\right),\left(u^{\prime}, v_{2}\right)$ and $\left(u^{\prime}, v_{3}\right)$ for every $v_{1} \in \mathbb{Z}_{q^{2}} \backslash\{0\}, u^{\prime}=t_{2} p_{2}, 1 \leq t_{2} \leq p_{1}-1$, $v_{2} \neq k q, 0 \leq k \leq q-1$ and $v_{3}=k q, 1 \leq k \leq q-1$. Therefore the degree of each such vertex is $q^{2}-1+p_{1}-1+\left(p_{1}-1\right)\left(q^{2}-q\right)+\left(p_{1}-1\right)(q-1)=p_{1} q^{2}-1$. Similarly if $u=t_{2} p_{2}, 1 \leq t_{2} \leq$ $p_{1}-1$ and $v=0$, then the degree of each such vertices is $p_{2} q^{2}-1$.

4. If $u=t_{1} p_{1}, 1 \leq t_{1} \leq p_{2}-1$ and $v \neq k q, 0 \leq k \leq q-1$, then each such vertex $(u, v)$ is connected to vertex $\left(u^{\prime}, 0\right)$ for every $u^{\prime}=t_{2} p_{2}, 1 \leq t_{2} \leq p_{1}-1$. Hence the degree of each such vertex is $p_{1}-1$. Similarly if $u=t_{2} p_{2}, 1 \leq t_{2} \leq p_{1}-1$ and $v \neq k q, 0 \leq k \leq q-1$, then the degree of each such vertex is $p_{2}-1$.

5. If $u=t_{1} p_{1}, 1 \leq t_{1} \leq p_{2}-1$ and $v=k q, 1 \leq k \leq q-1$, then each such vertex $(u, v)$ is connected to the vertices of type $\left(u^{\prime}, 0\right),\left(0, v^{\prime}\right)$ and $\left(u^{\prime}, v^{\prime}\right)$ for every $u^{\prime}=t_{2} p_{2}, 1 \leq t_{2} \leq p_{1}-1, v^{\prime}=k q, 1 \leq$ $k \leq q-1$. Therefore the degree of each such vertex is $p_{1}-1+q-1+\left(p_{1}-1\right)(q-1)=p_{1} q-1$. 
Similarly, if $u=t_{2} p_{2}, 1 \leq t_{2} \leq p_{1}-1$ and $v=k q, 1 \leq k \leq q-1$, then the degree of each such vertex is $p_{2} q-1$.

6. If $u \neq t_{1} p_{1}$ and $u \neq t_{2} p_{2}, 0 \leq t_{1} \leq p_{2}-1,0 \leq t_{2} \leq p_{1}-1$ and $v=k q, 1 \leq k \leq q-1$, then each such vertex $(u, v)$ is connected to the vertex $\left(0, v^{\prime}\right)$ for every $v^{\prime}=k q, 1 \leq k \leq q-1$. Hence the degree of each such vertex $(u, v)$ is $q-1$.

The total number of vertices of $\Gamma_{R}$ from the above cases is:

$$
\begin{aligned}
& q^{2}-q+\left(p_{2}-1\right)\left(p_{1}-1\right)+q-1+p_{2}-1+p_{1}-1+\left(p_{2}-1\right)\left(q^{2}-q\right)+\left(p_{1}-1\right)\left(q^{2}-q\right)+\left(p_{2}-1\right) \\
& (q-1)+\left(p_{1}-1\right)(q-1)+\left(p_{2}-1\right)\left(p_{1}-1\right)(q-1) \\
& =\left(q^{2}-1\right)\left(p_{2}+p_{1}-2\right)+p_{2} p_{1}+q^{2}-2+\left(p_{2}-1\right)\left(p_{1}-1\right)(q-1) \\
& =q^{2}\left(p_{2}+p_{1}-1\right)+q\left(p_{2}-1\right)\left(p_{1}-1\right)-1 .
\end{aligned}
$$

Hence, $\left|V\left(\Gamma_{R}\right)\right|=q^{2}\left(p_{2}+p_{1}-1\right)+q\left(p_{2}-1\right)\left(p_{1}-1\right)-1$.

The total number of edges of $\Gamma_{R}$ is obtained by using hand shaking lemma as:

$$
\begin{aligned}
& \left|E\left(\Gamma_{R}\right)\right|=\frac{\sum_{v \in V\left(\Gamma_{R}\right)} \operatorname{deg}(v)}{2}=\frac{1}{2}\left\{\left(q^{2}-q\right)\left(p_{1} p_{2}-1\right)+\left(p_{1}-1\right)\left(p_{2}-1\right)\left(q^{2}-1\right)+(q-1)\left(p_{1} p_{2} q-2\right)+\right. \\
& \left(p_{1}-1\right)\left(p_{2} q^{2}-1\right)+\left(p_{2}-1\right)\left(p_{1} q^{2}-1\right)+\left(p_{2}-1\right)\left(q^{2}-q\right)\left(p_{1}-1\right)+\left(p_{1}-1\right)\left(q^{2}-q\right)\left(p_{2}-1\right)+\left(p_{2}-\right. \\
& \left.1)(q-1)\left(p_{1} q-1\right)+\left(p_{1}-1\right)(q-1)\left(p_{2} q-1\right)+(q-1)^{2}\left(p_{1}-1\right)\left(p_{2}-1\right)\right\} .
\end{aligned}
$$

After simplification we get, $\left|E\left(\Gamma_{R}\right)\right|=q^{2}\left(5 p_{1} p_{2}-3 p_{1}-3 p_{2}+\frac{3}{2}\right)+q\left(-4 p_{1} p_{2}+2 p_{1}+2 p_{2}-\frac{3}{2}\right)+1$.

From the proof of Theorem 1 and our convenance, we partition the vertex set of $\Gamma_{R}$ as follows:

$$
\begin{aligned}
& \vee_{1}=\left\{(0, v): v \in \mathbb{Z}_{\left.q^{2}, v \neq k q, 0 \leq k \leq q-1\right\}}\right. \\
& \vee_{2}=\{(0, v): v=k q, 1 \leq k \leq q-1\} \\
& \vee_{3}=\left\{(u, 0): u=t_{1} p_{1}, 1 \leq t_{1} \leq p_{2}-1\right\} \\
& \vee_{4}=\left\{(u, 0): u=t_{2} p_{2}, 1 \leq t_{2} \leq p_{1}-1\right\} \\
& \vee_{5}=\left\{(u, 0): u \neq t_{1} p_{1}, u \neq t_{2} p_{2}, 1 \leq t_{1} \leq p_{2}-1,1 \leq t_{2} \leq p_{1}-1\right\} \\
& \vee_{6}=\left\{(u, v): u=t_{1} p_{1}, v \neq k q, 1 \leq t_{1} \leq p_{2}-1,0 \leq k \leq q-1\right\} \\
& \vee_{7}=\left\{(u, v): u=t_{2} p_{2}, v \neq k q, 1 \leq t_{2} \leq p_{1}-1,0 \leq k \leq q-1\right\} \\
& \vee_{8}=\left\{(u, v): u=t_{1} p_{1}, v=k q, 1 \leq t_{1} \leq p_{2}-1,1 \leq k \leq q-1\right\} \\
& \vee_{9}=\left\{(u, v): u=t_{2} p_{2}, v=k q, 1 \leq t_{2} \leq p_{1}-1,1 \leq k \leq q-1\right\} \\
& \vee_{10}=\left\{(u, v): u \neq t_{1} p_{1}, u \neq t_{2} p_{2}, v=k q, 1 \leq t_{1} \leq p_{2}-1,1 \leq t_{2} \leq p_{1}-1,1 \leq k \leq q-1\right\}
\end{aligned}
$$

This shows that $V\left(\Gamma_{R}\right)=\bigsqcup_{i=1}^{10} \vee_{i}$. It is observed that $\left|\vee_{1}\right|=q^{2}-q,\left|\vee_{2}\right|=q-1,\left|\vee_{3}\right|=p_{2}-1$, $\left|\vee_{4}\right|=p_{1}-1,\left|\vee_{5}\right|=\left(p_{1}-1\right)\left(p_{2}-1\right),\left|\vee_{6}\right|=\left(p_{2}-1\right)\left(q^{2}-q\right),\left|\vee_{7}\right|=\left(p_{1}-1\right)\left(q^{2}-q\right),\left|\vee_{8}\right|=$ $\left(p_{2}-1\right)(q-1),\left|\vee_{9}\right|=\left(p_{1}-1\right)(q-1)$ and $\left|\vee_{10}\right|=\left(p_{1}-1\right)\left(p_{2}-1\right)(q-1)$. Let the degree of a vertex $u$ in set $A$ be denoted by $d_{A}(u)$ and the distance between two vertices sets $X$ and $Y$ be $d(X, Y)=d(Y, X)$. Then following theorem determines the eccentricity of the vertices in graph $\Gamma_{R}$.

Theorem 2. If $p_{1}, p_{2}$ and $q$ are prime numbers such that $p_{1} \neq p_{2}$ and the associated zero divisor graph $\Gamma_{R}$ to the commutative ring $R=\mathbb{Z}_{p_{1} p_{2}} \times \mathbb{Z}_{q^{2}}$, then the eccentricity of the vertices of corresponding graph $\Gamma_{R}$ is either 2 or 3.

Proof. It is easy to see from the proof of Theorem 1 that, $d\left(\vee_{1}, \vee_{3}\right)=d\left(\vee_{1}, \vee_{4}\right)=d\left(\vee_{1}, \vee_{5}\right)=$ $d\left(\vee_{2}, \vee_{2}\right)=d\left(\vee_{2}, \vee_{3}\right)=d\left(\vee_{2}, \vee_{4}\right)=d\left(\vee_{2}, \vee_{5}\right)=d\left(\vee_{2}, \vee_{8}\right)=d\left(\vee_{2}, \vee_{9}\right)=d\left(\vee_{2}, \vee_{10}\right)=d\left(\vee_{3}, \vee_{4}\right)=$ $d\left(\vee_{3}, \vee_{7}\right)=d\left(\vee_{3}, \vee_{9}\right)=d\left(\vee_{4}, \vee_{6}\right)=d\left(\vee_{4}, \vee_{8}\right)=d\left(\vee_{8}, \vee_{9}\right)=1$ 


$$
\begin{gathered}
d\left(\vee_{1}, \vee_{2}\right)=d\left(\vee_{1}, \vee_{3}\right)+d\left(\vee_{3}, \vee_{2}\right)=1+1=2 \\
d\left(\vee_{1}, \vee_{6}\right)=d\left(\vee_{1}, \vee_{4}\right)+d\left(\vee_{4}, \vee_{6}\right)=1+1=2 \\
d\left(\vee_{1}, \vee_{7}\right)=d\left(\vee_{1}, \vee_{3}\right)+d\left(\vee_{3}, \vee_{7}\right)=1+1=2 \\
d\left(\vee_{1}, \vee_{8}\right)=d\left(\vee_{1}, \vee_{4}\right)+d\left(\vee_{4}, \vee_{8}\right)=1+1=2 \\
d\left(\vee_{1}, \vee_{9}\right)=d\left(\vee_{1}, \vee_{3}\right)+d\left(\vee_{3}, \vee_{9}\right)=1+1=2 \\
d\left(\vee_{1}, \vee_{10}\right)=d\left(\vee_{1}, \vee_{2}\right)+d\left(\vee_{2}, \vee_{10}\right)=2+1=3 \\
d\left(\vee_{1}, \vee_{1}\right)=d\left(\vee_{1}, \vee_{3}\right)+d\left(\vee_{3}, \vee_{1}\right)=1+1=2
\end{gathered}
$$

Hence the eccentricity of the corresponding vertices of set $\vee_{1}$ is 3 i.e., $\epsilon\left(\vee_{1}\right)=3$. Similarly, we can see that $\max \left\{d\left(\vee_{s}, \vee_{t}\right)\right\}=2$ for $s=2,3,4$ and $1 \leq t \leq 10$. Therefore, $\epsilon\left(\vee_{2}\right)=\epsilon\left(\vee_{3}\right)=\epsilon\left(\vee_{4}\right)=2$.

As $d\left(\vee_{5}, \vee_{6}\right)=d\left(\vee_{5}, \vee_{1}\right)+d\left(\vee_{1}, \vee_{6}\right)=1+2=3$ and $\max \left\{d\left(\vee_{5}, \vee_{t}\right)\right\}=3$ for $1 \leq t \leq 10$. This shows $\epsilon\left(\vee_{5}\right)=3$. Similarly, it is easy to calculate $\max \left\{d\left(\vee_{s}, \vee_{t}\right)\right\}=3$ for $s=6,7,8,9,10$ and $1 \leq t \leq 10$. This implies $\epsilon\left(\vee_{10}\right)=\epsilon\left(\vee_{9}\right)=\epsilon\left(\vee_{8}\right)=\epsilon\left(\vee_{7}\right)=\epsilon\left(\vee_{6}\right)=3$.

Lemma 1. For any three prime numbers $p_{1}, p_{2}$ and $q$ such that $p_{1} \neq p_{2}$ and the associated zero divisor graph $\Gamma_{R}$ of commutative ring $R=\mathbb{Z}_{p_{1} p_{2}} \times \mathbb{Z}_{q^{2}}$ we have,

$$
\begin{aligned}
& T\left(\Gamma_{R}\right)=\{\phi(2,2)+q \phi(2,3)\}(q-1)\left(p_{2}+p_{1}-2\right)+\left\{\phi(2,2)+\left(2 q^{2}+q-3\right) \phi(2,3)+\left(2 q^{2}-3 q+\right.\right. \\
& 1) \phi(3,3)\}\left(p_{2}-1\right)\left(p_{1}-1\right)+\left\{(q-2) \phi(2,2)+2(q-1)\left(p_{1} p_{2}-1\right) \phi(2,3)\right\} \frac{(q-1)}{2} .
\end{aligned}
$$

Proof. The graph $\Gamma_{R}$ contains $q^{2}\left(p_{2}+p_{1}-1\right)+q\left(p_{2}-1\right)\left(p_{1}-1\right)-1$ vertices and $q^{2}\left(5 p_{1} p_{2}-3 p_{1}-\right.$ $\left.3 p_{2}+\frac{3}{2}\right)+q\left(-4 p_{1} p_{2}+2 p_{1}+2 p_{2}-\frac{3}{2}\right)+1$ edges. Let us divide the edges of $\Gamma_{R}$ into partition sets according to the eccentricity of its end vertices.

$$
\Xi_{r, s}=\left\{u v \in E\left(\Gamma_{R}\right): \epsilon_{u}=r, \epsilon_{v}=s\right\}
$$

This means that the set $\Xi_{r, s}$ contains the edges incident with one vertex of eccentricity $r$ and the other vertex of eccentricity s. From the Theorem 1 and Theorem 2, we have $\left|\Xi_{2,2}\right|=\left(p_{2}+p_{1}-2\right)(q-1)+$ $\left(p_{2}-1\right)\left(p_{1}-1\right)+\frac{(q-1)(q-2)}{2},\left|\Xi_{2,3}\right|=2\left(q^{2}-1\right)\left(p_{2}-1\right)\left(p_{1}-1\right)+(q-1)^{2}\left(p_{1} p_{2}-1\right)+\left(q^{2}-q\right)\left(p_{2}+\right.$ $\left.p_{1}-2\right)+(q-1)\left(p_{2}-1\right)\left(p_{1}-1\right),\left|\Xi_{3,3}\right|=\left(p_{2}-1\right)\left(p_{1}-1\right)\left(2 q^{2}-3 q+1\right)$ and $E\left(\Gamma_{R}\right)=\Xi_{2,2} \cup \Xi_{2,3} \cup \Xi_{3,3}$. Then

$$
\begin{aligned}
T\left(G_{1}\right) & =\sum_{u v \in E\left(\Gamma_{R}\right)} \phi\left(\epsilon_{u}, \epsilon_{v}\right) \\
& =\sum_{u v \in \Xi_{2,2}} \phi(2,2)+\sum_{u v \in \Xi_{2,3}} \phi(2,3)+\sum_{u v \in \Xi_{3,3}} \phi(3,3) \\
& =\left(p_{2}+p_{1}-2\right)(q-1) \phi(2,2)+\left(p_{2}-1\right)\left(p_{1}-1\right) \phi(2,2)+\frac{(q-1)(q-2)}{2} \phi(2,2) \\
& +\left\{2\left(p_{2}-1\right)\left(p_{1}-1\right)\left(q^{2}-1\right)+(q-1)^{2}\left(p_{1} p_{2}-1\right)+\left(q^{2}-q\right)\left(p_{2}+p_{1}-2\right)\right\} \phi(2,3) \\
& +(q-1)\left(p_{2}-1\right)\left(p_{1}-1\right) \phi(2,3)+\left(p_{2}-1\right)\left(p_{1}-1\right)\left(2 q^{2}-3 q+1\right) \phi(3,3) \\
& =\{\phi(2,2)+q \phi(2,3)\}(q-1)\left(p_{2}+p_{1}-2\right)+\left\{(q-2) \phi(2,2)+2(q-1)\left(p_{1} p_{2}-1\right) \phi(2,3)\right\} \frac{(q-1)}{2} \\
& +\left\{\phi(2,2)+\left(2 q^{2}+q-3\right) \phi(2,3)+\left(2 q^{2}-3 q+1\right) \phi(3,3)\right\}\left(p_{2}-1\right)\left(p_{1}-1\right) .
\end{aligned}
$$

Now we present values of the eccentric topological indices of the graph $\Gamma_{R}$ which are edge-based. 
Theorem 3. If $p_{1}, p_{2}$ and $q$ are prime numbers such that $p_{1} \neq p_{2}$ and the associated zero divisor graph $\Gamma_{R}$ to the commutative ring $R=\mathbb{Z}_{p_{1} p_{2}} \times \mathbb{Z}_{q^{2}}$, then

the First Zagreb eccentricity index of $\Gamma_{R}$ is:

$M_{1}^{*}\left(\Gamma_{R}\right)=(5 q+4)(q-1)\left(p_{2}+p_{1}-2\right)+\left(10 p_{1} p_{2} q-10 p_{1} p_{2}-6 q+2\right) \frac{q-1}{2}+\left(22 q^{2}-13 q-5\right)\left(p_{2}-\right.$ 1) $\left(p_{1}-1\right)$,

the third Zagreb eccentricity index is:

$M_{3}^{*}\left(\Gamma_{R}\right)=(6 q+4)(q-1)\left(p_{2}+p_{1}-2\right)+2(q-1)\left(3 p_{1} p_{2} q-3 p_{1} p_{2}-2 q+1\right)+\left(30 q^{2}-21 q-5\right)\left(p_{2}-\right.$ 1) $\left(p_{1}-1\right)$,

the geometric-arithmetic eccentricity index is:

$G A_{4}\left(\Gamma_{R}\right)=\left(\frac{2 \sqrt{6}}{5} q+1\right)(q-1)\left(p_{2}+p_{1}-2\right)+\frac{(q-1)(q-2)}{2}+\frac{2 \sqrt{6}}{5}(q-1)^{2}\left(p_{1} p_{2}-1\right)+\left(\frac{4 \sqrt{6}+10}{5} q^{2}+\right.$ $\left.\frac{2 \sqrt{6}-15}{5} q+\frac{10-6 \sqrt{6}}{5}\right)\left(p_{2}-1\right)\left(p_{1}-1\right)$,

the atom-bond connectivity eccentricity index is:

$A B C_{5}\left(\Gamma_{R}\right)=\frac{\sqrt{2}}{2}\left(q^{2}-1\right)\left(p_{2}+p_{1}-2\right)+\frac{\sqrt{2}(q-1)}{4}\left(2 p_{1} p_{2} q-2 p_{1} p_{2}-q\right)+\left(\frac{3 \sqrt{2}+4}{3} q^{2}+\frac{\sqrt{2}-4}{2} q+\right.$ $\left.\frac{2-3 \sqrt{2}}{3}\right)\left(p_{2}-1\right)\left(p_{1}-1\right)$,

the fourth type of eccentric harmonic index is:

$H_{4}\left(\Gamma_{R}\right)=\frac{4 q+5}{10}(q-1)\left(p_{2}+p_{1}-2\right)+\frac{(q-1)}{2}\left(\frac{4}{5} p_{1} p_{2} q-\frac{4}{5} p_{1} p_{2}-\frac{3}{10} q-\frac{1}{5}\right)+\left(\frac{22}{15} q^{2}-\frac{3}{5} q-\frac{11}{30}\right)\left(p_{2}-\right.$ 1) $\left(p_{1}-1\right)$.

Proof. For the first Zagreb eccentricity index $M_{1}^{*}\left(\Gamma_{R}\right)$ of $\Gamma_{R}$ we obtain $\phi\left(\epsilon_{u}, \epsilon_{v}\right)=\epsilon_{u}+\epsilon_{v}$. So $\phi(2,2)=4$, $\phi(2,3)=5$ and $\phi(3,3)=6$. Thus by Lemma 1 ,

$$
\begin{aligned}
M_{1}^{*}\left(\Gamma_{R}\right) & =(4+5 q)(q-1)\left(p_{2}+p_{1}-2\right)+\left(4(q-2)+10(q-1)\left(p_{1} p_{2}-1\right)\right) \frac{q-1}{2} \\
& +\left(4+5\left(2 q^{2}+q-3\right)+6\left(2 q^{2}-3 q+1\right)\right)\left(p_{2}-1\right)\left(p_{1}-1\right) \\
& =(5 q+4)(q-1)\left(p_{2}+p_{1}-2\right)+\left(10 p_{1} p_{2} q-10 p_{1} p_{2}-6 q+2\right) \frac{q-1}{2} \\
& +\left(22 q^{2}-13 q-5\right)\left(p_{2}-1\right)\left(p_{1}-1\right) .
\end{aligned}
$$

For the third Zagreb eccentricity index $M_{3}^{*}\left(\Gamma_{R}\right)$ of $\Gamma_{R}$ we obtain $\phi\left(\epsilon_{u}, \epsilon_{v}\right)=\epsilon_{u} \times \epsilon_{v}$. So $\phi(2,2)=4$, $\phi(2,3)=6$ and $\phi(3,3)=9$ So by Lemma 1 ,

$$
\begin{aligned}
M_{3}^{*}\left(\Gamma_{R}\right) & =(4+6 q)(q-1)\left(p_{2}+p_{1}-2\right)+\left(4(q-2)+12(q-1)\left(p_{1} p_{2}-1\right)\right) \frac{q-1}{2} \\
& +\left(4+6\left(2 q^{2}+q-3\right)+9\left(2 q^{2}-3 q+1\right)\right)\left(p_{2}-1\right)\left(p_{1}-1\right) \\
& =(6 q+4)(q-1)\left(p_{2}+p_{1}-2\right)+2(q-1)\left(3 p_{1} p_{2} q-3 p_{1} p_{2}-2 q+1\right) \\
& +\left(30 q^{2}-21 q-5\right)\left(p_{2}-1\right)\left(p_{1}-1\right) .
\end{aligned}
$$


For the geometric-arithmetic eccentricity index $G A_{4}\left(\Gamma_{R}\right)$ of $\Gamma_{R}$, we get $\phi\left(\epsilon_{u}, \epsilon_{v}\right)=\frac{2 \sqrt{\epsilon_{u} \times \epsilon_{v}}}{\epsilon_{u}+\epsilon_{v}}$. So $\phi(2,2)=1, \phi(2,3)=\frac{2 \sqrt{6}}{5}$ and $\phi(3,3)=1$. Therefore,

$$
\begin{aligned}
G A_{4}\left(\Gamma_{R}\right) & =\left(1+\frac{2 \sqrt{6}}{5} q\right)(q-1)\left(p_{2}+p_{1}-2\right)+\left((q-2)+2(q-1)\left(p_{1} p_{2}-1\right) \frac{2 \sqrt{6}}{5}\right) \frac{q-1}{2} \\
& +\left(1+\frac{2 \sqrt{6}}{5}\left(2 q^{2}+q-3\right)+\left(2 q^{2}-3 q+1\right)\right)\left(p_{2}-1\right)\left(p_{1}-1\right) \\
& =\left(\frac{2 \sqrt{6}}{5} q+1\right)(q-1)\left(p_{2}+p_{1}-2\right)+\frac{(q-1)(q-2)}{2}+\frac{2 \sqrt{6}}{5}(q-1)^{2}\left(p_{1} p_{2}-1\right) \\
& +\left(\frac{4 \sqrt{6}+10}{5} q^{2}+\frac{2 \sqrt{6}-15}{5} q+\frac{10-6 \sqrt{6}}{5}\right)\left(p_{2}-1\right)\left(p_{1}-1\right) .
\end{aligned}
$$

For the atom-bond connectivity eccentricity index $A B C_{5}\left(\Gamma_{R}\right)$ of $\Gamma_{R}$, we obtain $\phi\left(\epsilon_{u}, \epsilon_{v}\right)=\sqrt{\frac{\epsilon_{u}+\epsilon_{v}-2}{\epsilon_{u} \times \epsilon_{v}}}$. Thus $\phi(2,2)=\phi(2,3)=\frac{1}{\sqrt{2}}$ and $\phi(3,3)=\frac{2}{3}$ Therefore,

$$
\begin{aligned}
A B C_{5}\left(\Gamma_{R}\right) & =\left(\frac{1}{\sqrt{2}}+\frac{1}{\sqrt{2}} q\right)(q-1)\left(p_{2}+p_{1}-2\right)+\left(\frac{1}{\sqrt{2}}(q-2)+2(q-1)\left(p_{1} p_{2}-1\right) \frac{1}{\sqrt{2}}\right) \frac{q-1}{2} \\
& +\left(\frac{1}{\sqrt{2}}+\frac{1}{\sqrt{2}}\left(2 q^{2}+q-3\right)+\frac{2}{3}\left(2 q^{2}-3 q+1\right)\right)\left(p_{2}-1\right)\left(p_{1}-1\right) \\
& =\frac{\sqrt{2}}{2}\left(q^{2}-1\right)\left(p_{2}+p_{1}-2\right)+\frac{\sqrt{2}(q-1)}{4}\left(2 p_{1} p_{2} q-2 p_{1} p_{2}-q\right) \\
& +\left(\frac{3 \sqrt{2}+4}{3} q^{2}+\frac{\sqrt{2}-4}{2} q+\frac{2-3 \sqrt{2}}{3}\right)\left(p_{2}-1\right)\left(p_{1}-1\right) .
\end{aligned}
$$

For the fourth type of eccentric harmonic index $H_{4}\left(\Gamma_{R}\right)$ of $\Gamma_{R}$, we get $\phi\left(\epsilon_{u}, \epsilon_{v}\right)=\frac{2}{\epsilon_{u}+\epsilon_{v}}$, therefore $\phi(2,2)=\frac{1}{2}, \phi(2,3)=\frac{2}{5}$ and $\phi(3,3)=\frac{1}{3}$. Thus,

$$
\begin{aligned}
H_{4}\left(\Gamma_{R}\right) & =\left(\frac{1}{2}+\frac{2}{5} q\right)(q-1)\left(p_{2}+p_{1}-2\right)+\left(\frac{1}{2}(q-2)+2(q-1)\left(p_{1} p_{2}-1\right) \frac{2}{5}\right) \frac{q-1}{2} \\
& +\left(\frac{1}{2}+\frac{2}{5}\left(2 q^{2}+q-3\right)+\frac{1}{3}\left(2 q^{2}-3 q+1\right)\right)\left(p_{2}-1\right)\left(p_{1}-1\right) \\
& =\frac{4 q+5}{10}(q-1)\left(p_{2}+p_{1}-2\right)+\frac{(q-1)}{2}\left(\frac{4}{5} p_{1} p_{2} q-\frac{4}{5} p_{1} p_{2}-\frac{3}{10} q-\frac{1}{5}\right) \\
& +\left(\frac{22}{15} q^{2}-\frac{3}{5} q-\frac{11}{30}\right)\left(p_{2}-1\right)\left(p_{1}-1\right) .
\end{aligned}
$$

5.2. Case 2: $\mathbb{Z}_{p^{2}} \times \mathbb{Z}_{q^{2}}$

In this subsection, we discuss the eccentric topological indices for zero divisor graph $Y_{R}$ defined on commutative ring $R=\mathbb{Z}_{p^{2}} \times \mathbb{Z}_{q^{2}}$, where $p, q$ are prime numbers.

Theorem 4. Let $p$ and $q$ be prime numbers and $Y_{R}$ be the zero divisor graph of commutative ring $R=$ $\mathbb{Z}_{p^{2}} \times \mathbb{Z}_{q^{2}}$. Then $\left|V\left(\mathrm{Y}_{R}\right)\right|=p^{2} q+p q^{2}-p q-1$ and $\left|E\left(\mathrm{Y}_{R}\right)\right|=\frac{(p-1)(q-1)(5 p q-2)+2 p^{2} q^{2}-p^{2} q-p q^{2}-2 p-2 q+4}{2}$.

Proof. For any two prime numbers $p$ and $q$, we consider the ring $R=\mathbb{Z}_{p^{2}} \times \mathbb{Z}_{q^{2}}$ with usual operations. The associated zero divisor graph $\mathrm{Y}_{R}$ on ring $R$ is defined as: Any $(u, v) \in V\left(\mathrm{Y}_{R}\right)$ if and only if the elements $u=k p, 0 \leq k \leq p-1$ or $v=t q, 0 \leq t \leq q-1$ with $(u, v) \neq(0,0)$. Therefore, a non zero 
element $(u, v)$ is not a zero divisor in the ring $R$ if and only if $u$ is not a multiple of $p$ and $v$ is not a multiple of $q$. As the number of elements in $\mathbb{Z}_{p^{2}}$ which are not a multiple of $p$ are $p^{2}-p$ and the number of elements in $\mathbb{Z}_{q^{2}}$ which are not a multiple of $q$ are $q^{2}-q$. Also, the element $(0,0)$ is not a zero divisor in $R$. Thus the total number of non zero divisors in the ring $R$ is $\left(p^{2}-p\right)\left(q^{2}-q\right)+1$. This implies

$$
\left|V\left(\mathrm{Y}_{R}\right)\right|=p^{2} q^{2}-\left(\left(p^{2}-p\right)\left(q^{2}-q\right)+1\right)=p^{2} q+p q^{2}-p q-1
$$

For any $(u, v) \in V\left(\mathrm{Y}_{R}\right)$, we discuss the degree of each vertex as follows:

1. If $u=0$ and $v$ is not a multiple of $q$, then vertex $(0, v)$ of this form is connected to each vertex $\left(u^{\prime}, 0\right)$, where $u^{\prime} \in \mathbb{Z}_{p^{2}} \backslash\{0\}$. Hence each vertex $(0, v)$ has degree $p^{2}-1$. By symmetry each vertex $(u, 0)$ such that $u$ is not a multiple of $p$ has degree $q^{2}-1$.

2. If $u=0$ and $v \in\{q, 2 q, \ldots,(q-1) q\}$, then vertex $(0, v)$ of this form is connected to each vertex $\left(u^{\prime}, v^{\prime}\right)$, where $u^{\prime} \in \mathbb{Z}_{p^{2}}$ and $v^{\prime} \in\{0, q, 2 q, \ldots,(q-1) q\}$ with $\left(u^{\prime}, v^{\prime}\right) \notin\{(0,0),(0, v)\}$. Hence the each vertex of this type $(0, v)$ has degree $p^{2} q-2$. By symmetry the degree of each vertex $(u, 0)$ such that $u \in\{p, 2 p, \ldots,(p-1) p\}$ is $p q^{2}-2$.

3. If $u$ is not a multiple of $p$ and $v \in\{q, 2 q, \ldots,(q-1) q\}$, then each vertex $(u, v)$ is connected to each vertex $\left(0, v^{\prime}\right), v^{\prime} \in\{q, 2 q, \ldots,(q-1) q\}$. Hence each vertex $(u, v)$ of this type has degree $q-1$. By symmetry the degree of each vertex $(u, v)$ if $u \in\{p, 2 p, \ldots,(p-1) p\}$ and $v$ is not a multiple of $q$ is $p-1$.

4. If $u \in\{p, 2 p, \ldots,(p-1) p\}$ and $v \in\{q, 2 q, \ldots,(q-1) q\}$, then each vertex $(u, v)$ of this form is connected to each vertex $\left(u^{\prime}, v^{\prime}\right)$, where $u^{\prime} \in\{0, p, 2 p, \ldots,(p-1) p\}$ and $v^{\prime} \in\{0, q, 2 q, \ldots,(q-$ 1) $q\}$ with $\left(u^{\prime}, v^{\prime}\right) \notin\{(0,0),(0, v)\}$. Hence the degree of each vertex $(u, v)$ is $p q-2$.

From the above discussion, it is easy to see that the zero divisor graph $Y_{R}$ contains $q^{2}-q$ vertices of degree $p^{2}-1, p^{2}-p$ vertices of degree $q^{2}-1, p-1$ vertices of degree $p q^{2}-2, q-1$ vertices of degree $q p^{2}-2, q(p-1)(q-1)$ vertices of degree $p-1, p(p-1)(q-1)$ vertices of degree $q-1$ and $(p-1)(q-1)$ vertices of degree $p q-2$. By using the hand shaking lemma the number of edges of $\mathrm{Y}_{R}$ are $\left|E\left(\mathrm{Y}_{R}\right)\right|=\frac{1}{2}\left(\left(p^{2}-1\right)\left(q^{2}-q\right)+\left(q^{2}-1\right)\left(p^{2}-p\right)+\left(p q^{2}-2\right)(p-1)+\right.$ $\left.\left(q p^{2}-2\right)(q-1)+(p-1) q(p-1)(q-1)+(q-1) p(p-1)(q-1)+(p q-2)(p-1)(q-1)\right)=$ $\frac{(p-1)(q-1)(5 p q-2)+2 p^{2} q^{2}-p^{2} q-p q^{2}-2 p-2 q+4}{2}$.

Let $\Im_{i}$ denotes the set of containing the vertices of degree $i$. From the proof of Theorem 4 , we get $\left|\Im_{p^{2}-1}\right|=q^{2}-q,\left|\Im_{q^{2}-1}\right|=p^{2}-p,\left|\Im_{p q^{2}-2}\right|=p-1,\left|\Im_{q p^{2}-2}\right|=q-1,\left|\Im_{p-1}\right|=$ $q(p-1)(q-1),\left|\Im_{q-1}\right|=p(p-1)(q-1)$ and $\left|\Im_{p q-2}\right|=(p-1)(q-1)$. The following theorem determines the eccentricity of the vertices in graph of $\mathrm{Y}_{R}$.

Theorem 5. Let $p$ and $q$ be two prime numbers and $Y_{R}$ be the zero divisor graph of commutative ring $R=\mathbb{Z}_{p^{2}} \times \mathbb{Z}_{q^{2}}$. Then the eccentricity of a vertex of $Y_{R}$ is either 2 or 3 .

Proof. Similar proof of Theorem 2, we get $\epsilon\left(\Im_{p q^{2}-2}\right)=\epsilon\left(\Im_{q p^{2}-2}\right)=\epsilon\left(\Im_{p q-2}\right)=2$ and $\epsilon\left(\Im_{p^{2}-1}\right)=$ $\epsilon\left(\Im_{q^{2}-1}\right)=\epsilon\left(\Im_{p-1}\right)=\epsilon\left(\Im_{q-1}\right)=3$.

Lemma 2. Let $p$ and $q$ be two prime numbers and $Y_{R}$ be the zero divisor graph of commutative ring $R=$ $\mathbb{Z}_{p^{2}} \times \mathbb{Z}_{q^{2}}$. Then

$$
T\left(\mathrm{Y}_{R}\right)=\frac{p^{2} q+p q^{2}-4 p q+2}{2} \phi(2,2)+p q(p-1)(q-1)\left(\frac{\phi(2,2)}{2}+2 \phi(2,3)+\phi(3,3)\right) .
$$

Proof. The graph $\mathrm{Y}_{R}$ contains $p^{2} q+p q^{2}-p q-1$ vertices and $\frac{(p-1)(q-1)(5 p q-2)+2 p^{2} q^{2}-p^{2} q-p q^{2}-2 p-2 q+4}{2}$ edges. Let us divide the edges of $Y_{R}$ into partition sets with respect to the degree of endpoints of each edge as: 


$$
\Xi_{r, s}=\left\{u v \in E\left(\mathrm{Y}_{R}\right): \epsilon_{u}=r, \epsilon_{v}=s\right\} .
$$

This means that the set $\Xi_{r, S}$ contains the edges incident with one vertex of eccentricity $r$ and the other vertex of eccentricity $s$. From the Theorem 4 and Theorem 5, we get $\left|\Xi_{2,2}\right|=\frac{(p-1)(p-2)}{2}+$ $\frac{(q-1)(q-2)}{2}+\frac{(p-1)(q-1)(p q+p+q-2)}{2},\left|\Xi_{2,3}\right|=2 p q(p-1)(q-1),\left|\Xi_{3,3}\right|=p q(p-1)(q-1)$ and $E\left(\mathrm{Y}_{R}\right)=$ $\Xi_{2,2} \cup \Xi_{2,3} \cup \Xi_{3,3}$. Then

$$
\begin{aligned}
T\left(\mathrm{Y}_{R}\right) & =\sum_{u v \in E\left(G_{R}\right)} \phi\left(\epsilon_{u}, \epsilon_{v}\right) \\
& =\sum_{u v \in \Xi_{2,2}} \phi(2,2)+\sum_{u v \in \Xi_{2,3}} \phi(2,3)+\sum_{u v \in \Xi_{3,3}} \phi(3,3) \\
& =\left\{\frac{(p-1)(p-2)}{2}+\frac{(q-1)(q-2)}{2}+\frac{(p-1)(q-1)(p q+p+q-2)}{2}\right\} \phi(2,2) \\
& +2 p q(p-1)(q-1) \phi(2,3)+p q(p-1)(q-1) \phi(3,3) \\
& =\frac{p^{2} q+p q^{2}-4 p q+2}{2} \phi(2,2)+p q(p-1)(q-1)\left(\frac{\phi(2,2)}{2}+2 \phi(2,3)+\phi(3,3)\right) .
\end{aligned}
$$

Now we present values of eccentric topological indices based on edges of graph $\mathrm{Y}_{R}$.

Theorem 6. Let $p$ and $q$ be two prime numbers and $Y_{R}$ be the zero divisor graph of commutative ring $R=\mathbb{Z}_{p^{2}} \times \mathbb{Z}_{q^{2}}$. Then the First Zagreb eccentricity index of $Y_{R}$ is:

$$
M_{1}^{*}\left(\mathrm{Y}_{R}\right)=2\left(p^{2} q+p q^{2}-4 p q+2\right)+18 p q(p-1)(q-1)
$$

the third Zagreb eccentricity index is:

$$
M_{3}^{*}\left(\mathrm{Y}_{R}\right)=2\left(p^{2} q+p q^{2}-4 p q+2\right)+23 p q(p-1)(q-1)
$$

the geometric-arithmetic eccentricity index is:

$$
G A_{4}\left(\mathrm{Y}_{R}\right)=\frac{p^{2} q+p q^{2}-4 p q+2}{2}+p q(p-1)(q-1)\left(\frac{15+8 \sqrt{6}}{10}\right)
$$

the atom-bond connectivity eccentricity index is:

$$
A B C_{5}\left(\mathrm{Y}_{R}\right)=\frac{p^{2} q+p q^{2}-4 p q+2}{2 \sqrt{2}}+p q(p-1)(q-1)\left(\frac{15+4 \sqrt{2}}{6 \sqrt{2}}\right)
$$

the fourth type of eccentric harmonic index is:

$$
H_{4}\left(\mathrm{Y}_{R}\right)=\frac{p^{2} q+p q^{2}-4 p q+2}{4}+\frac{83}{60} p q(p-1)(q-1) .
$$

Proof. For the first Zagreb eccentricity index $M_{1}^{*}\left(\mathrm{Y}_{R}\right)$ of $\mathrm{Y}_{R}$ we obtain $\phi\left(\epsilon_{u}, \epsilon_{v}\right)=\epsilon_{u}+\epsilon_{v}$. So $\phi(2,2)=4$, $\phi(2,3)=5$ and $\phi(3,3)=6$. Thus by Lemma 2 ,

$$
\begin{aligned}
M_{1}^{*}\left(\mathrm{Y}_{R}\right) & =\frac{p^{2} q+p q^{2}-4 p q+2}{2} \phi(2,2)+p q(p-1)(q-1)\left(\frac{\phi(2,2)}{2}+2 \phi(2,3)+\phi(3,3)\right) \\
& =2\left(p^{2} q+p q^{2}-4 p q+2\right)+18 p q(p-1)(q-1) .
\end{aligned}
$$

For the third Zagreb eccentricity index $M_{3}^{*}\left(Y_{R}\right)$ of $Y_{R}$ we obtain $\phi\left(\epsilon_{u}, \epsilon_{v}\right)=\epsilon_{u} \times \epsilon_{v}$. So $\phi(2,2)=4$, $\phi(2,3)=6$ and $\phi(3,3)=9$ So by Lemma 2 , 


$$
\begin{aligned}
M_{3}^{*}\left(\mathrm{Y}_{R}\right) & =\frac{p^{2} q+p q^{2}-4 p q+2}{2} \phi(2,2)+p q(p-1)(q-1)\left(\frac{\phi(2,2)}{2}+2 \phi(2,3)+\phi(3,3)\right) \\
& =2\left(p^{2} q+p q^{2}-4 p q+2\right)+23 p q(p-1)(q-1) .
\end{aligned}
$$

For the geometric-arithmetic eccentricity index $G A_{4}\left(\mathrm{Y}_{R}\right)$ of $\mathrm{Y}_{R}$, we get $\phi\left(\epsilon_{u}, \epsilon_{v}\right)=\frac{2 \sqrt{\epsilon_{u} \times \epsilon_{v}}}{\epsilon_{u}+\epsilon_{v}}$. So $\phi(2,2)=1, \phi(2,3)=\frac{2 \sqrt{6}}{5}$ and $\phi(3,3)=1$. Therefore,

$$
\begin{aligned}
G A_{4}\left(\mathrm{Y}_{R}\right) & =\frac{p^{2} q+p q^{2}-4 p q+2}{2} \phi(2,2)+p q(p-1)(q-1)\left(\frac{\phi(2,2)}{2}+2 \phi(2,3)+\phi(3,3)\right) \\
& =\frac{p^{2} q+p q^{2}-4 p q+2}{2}+p q(p-1)(q-1)\left(\frac{15+8 \sqrt{6}}{10}\right) .
\end{aligned}
$$

For the atom-bond connectivity eccentricity index $A B C_{5}\left(\mathrm{Y}_{R}\right)$ of $\mathrm{Y}_{R}$, we obtain $\phi\left(\epsilon_{u}, \epsilon_{v}\right)=\sqrt{\frac{\epsilon_{u}+\epsilon_{v}-2}{\epsilon_{u} \times \epsilon_{v}}}$. Thus $\phi(2,2)=\phi(2,3)=\frac{1}{\sqrt{2}}$ and $\phi(3,3)=\frac{2}{3}$ Therefore,

$$
\begin{aligned}
A B C_{5}\left(\mathrm{Y}_{R}\right) & =\frac{p^{2} q+p q^{2}-4 p q+2}{2} \phi(2,2)+p q(p-1)(q-1)\left(\frac{\phi(2,2)}{2}+2 \phi(2,3)+\phi(3,3)\right) \\
& =\frac{p^{2} q+p q^{2}-4 p q+2}{2 \sqrt{2}}+p q(p-1)(q-1)\left(\frac{15+4 \sqrt{2}}{6 \sqrt{2}}\right)
\end{aligned}
$$

For the fourth type of eccentric harmonic index $H_{4}\left(\mathrm{Y}_{R}\right)$ of $\mathrm{Y}_{R}$, we get $\phi\left(\epsilon_{u}, \epsilon_{v}\right)=\frac{2}{\epsilon_{u}+\epsilon_{v}}$, therefore $\phi(2,2)=\frac{1}{2}, \phi(2,3)=\frac{2}{5}$ and $\phi(3,3)=\frac{1}{3}$. Thus,

$$
\begin{aligned}
H_{4}\left(\mathrm{Y}_{R}\right) & =\frac{p^{2} q+p q^{2}-4 p q+2}{2} \phi(2,2)+p q(p-1)(q-1)\left(\frac{\phi(2,2)}{2}+2 \phi(2,3)+\phi(3,3)\right) \\
& =\frac{p^{2} q+p q^{2}-4 p q+2}{4}+\frac{83}{60} p q(p-1)(q-1) .
\end{aligned}
$$

\section{Conclusions}

This article emphasizes the computation of first Zagreb eccentricity index, third Zagreb eccentricity index, geometric-arithmetic eccentricity index, atom-bond connectivity eccentricity index and the fourth type of eccentric harmonic index for zero divisor graphs associated with algebraic structures that are commutative rings $\mathbb{Z}_{p_{1} p_{2}} \times \mathbb{Z}_{q^{2}}$ and $\mathbb{Z}_{p^{2}} \times \mathbb{Z}_{q^{2}}$. This study will be useful for the further comprehension of the attributes of various physical structures like carbohydrates, silicone structures, polymers, hexagonal chains and cylindrical fullerenes. Likewise, they can be used for creating a productive physical structure in mechanics as well as for different computer network problems.

Author Contributions: A.N.A.K. contribute for Conceptualization, Data curation, Funding acquisition, Project administration and Resources. A.A. contribute for Investigation, Methodology, Supervision, and Validation. A.H. contribute for Formal analysis, Project administration, and Visualization. All authors read and approved the initial and final version of the paper.

Funding: This research received no external funding.

Conflicts of Interest: The authors declare no conflict of interest.

\section{References}

1. Ahmad, A.; Haider, A. Computing the radio labeling associated with zero divisor graph of a commutative ring. UPB Sci. Bull. Ser. A 2019, 81, 65-72.

2. Beck, I. Coloring of a commutative ring. J. Algebra 1988, 116, 208-226. [CrossRef] 
3. Bača, M.; Horvràthovà, J.; Mokrišovà, M.; Suhànyiovà, A. On topological indices of fullerenes. Appl. Math . Comput. 2015, 251, 154-161.

4. Gutman, I.; Polansky, O.E. Mathematical Concepts in Organic Chemistry; Springer-Verlag: New York, NY, USA, 1986.

5. Ahmad, A. On the degree based topological indices of benzene ring embedded in P-type-surface in 2D network. Hacet. J. Math. Stat. 2018, 47, 9-18. [CrossRef]

6. Ahmad, A. Computation of certain topological properties of honeycomb networks and Graphene. Discret. Math. Algorithms Appl. (DMAA) 2017, 9, 1750064. [CrossRef]

7. Akhter, S.; Gao, W.; Imran, M.; Farahani, M.R. On topological indices of honeycomb networks and graphene networks. Hacet. J. Math. Stat. 2018, 47, 19-35. [CrossRef]

8. Nadeem, M.F.; Zafar, S.; Zahid, Z. On topological properties of the line graphs of subdivision graphs of certain nanostructures. Appl. Math. Comput. 2016, 273, 125-130. [CrossRef]

9. Wang, S.; Farahani, M.R.; Kanna, M.R.R.; Jamil, M.K.; Kumar, R.P. The Wiener Index and the Hosoya Polynomial of the Jahangir Graphs. Appl. Comput. Math. 2016, 5, 138-141. [CrossRef]

10. Wiener, H. Structural determination of paraffin boiling points. J. Am. Chem. Soc. 1947, 69, 17-20. [CrossRef] [PubMed]

11. Ghorbani, M.; Hosseinzadeh, M.A. A new version of Zagreb indices. Filomat 2012, 26, 93-100. [CrossRef]

12. Vukičević, D.; Graovac, A. Note on the comparison of the first and second normalized Zagreb eccentricity indices. Acta Chim. Slov. 2010, 57, 524-528. [PubMed]

13. Ghorbani, M.; Khaki, A. A note on the fourth version of geometric-arithmetic index. Optoelectron. Adv. Mater. Rapid Commum. 2010, 4, 2212-2215.

14. Farahani, M.R. Eccentricity version of atom bond connectivity index of benzenoid family $A B C_{5}\left(\mathrm{Hk}_{\mathrm{k}}\right.$. World Appl. Sci. J. Chem. 2013, 21, 1260-1265.

15. Farahani, M.R.; Ediz, S.; Imran, M. On novel harmonic indices of certain nanotubes. Int. J. Adv. Biotechnol. Res. 2017, 8, 277-282.

16. Gao, Y.; Ediz, S.; Farahani, M.R.; Imran, M. On the Second Harmonic Index of Titania Nanotubes. Drug Des. Int. Prop. Int. J. 2018, 1, 7-10.

17. Estrada, E.; Torres, L.; Rodriguez, L.; Gutman, I. An atom-bond connectivity index: modelling the enthalpy of formation of alkanes. Indian J. Chem. 1998, 37A, 849-855.

18. Siddiqui, M.K.; Naeem, M.; Rehman, N.A.; Imran, M. Computing topological indices of certain networks. J. Optoelectron. Adv. Mater. 2016, 18, 884-892.

19. Shao, Z.; Wu, P.; Gao, Y.; Gutman, I.; Zhang, X. On the maximum $A B C$ index of graphs without pendent vertices. Appl. Math. Comput. 2017, 315, 298-312. [CrossRef]

20. Siddiqui, M.K.; Imran, M.; Ahmad, A. On Zagreb indices, Zagreb polynomials of some nanostar dendrimers. Appl. Math. Comput. 2016, 280, 132-139. [CrossRef]

21. Shao, Z.; Siddiqui, M.K.; Muhammad, M.H. Computing zagreb indices and zagreb polynomials for symmetrical nanotubes. Symmetry 2018, 10, 244. [CrossRef]

22. Gupta, S.; Singh, M.; Madan, A.K. Application of Graph Theory: Relationship of Eccentric Connectivity Index and Wiener's Index with Anti-inflammatory Activity. J. Math. Anal. Appl. 2002, 266, 259-268. [CrossRef]

23. Elahi, K.; Ahmad, A.; Hasni, R. Construction algorithm for zero divisor graphs of finite commutative rings and their vertex-based eccentric topological indices. Mathematics 2018, 6, 301. [CrossRef]

24. Imran, M.; Siddiqui, M.K.; Abunamous, A.A.E.; Adi, D.; Rafique, S.H.; Baig, A.Q. Eccentricity based topological indices of an oxide network. Mathematics 2018, 6, 126. [CrossRef]

25. Harris, J.M.; Hirst, J.L.; Mossinghoff, M.J. Combinatorics and Graph Theory; Springer Science and Business Media: Berlin, Germany, 2008.

26. Anderson, D.F.; Livingston, P.S. The Zero-divisor Graph of Commutative Ring. J. Algebra 1999, 217, 434-447. [CrossRef]

27. Akbari, S.; Mohammadian, A. On the zero-divisor graph of a commutative ring. J. Algebra 2004, 274, 847-855. [CrossRef]

28. Anderson, D.F.; Mulay, S.B. On the diameter and girth of a zero-divisor graph. J. Pure Appl. Algebra 2008, 210, 543-550. [CrossRef]

(c) 2019 by the authors. Licensee MDPI, Basel, Switzerland. This article is an open access article distributed under the terms and conditions of the Creative Commons Attribution (CC BY) license (http://creativecommons.org/licenses/by/4.0/). 